\title{
LISTE DES RELECTEURS POUR LES ANNÉES 2013 ET 2014
}

Nous remercions très chaleureusement les relecteurs suivants pour le temps et l'énergie qu'ils ont donné à la Revue Française de Photogrammétrie et de Télédétection.

- Mohamed Ait Kbir

- Pascal Allemand

- Amina Amharef

- Alice Andral

- Jean-Stéphane Bailly

- Nicolas Barbier

- Christian Baron

- Stéphanie Battiston

- Agnès Bégué

- Thierry Belouard

- Etienne Berthier

- Patrice Bicheron

- Clélia Bilodeau

- Hélène de Boissezon

- Mathieu Brédif

- Elodie Buard

- Jean-Philippe Cantou

- Nicolas Champion

- Laure Chandelier

- Nesrine Chehata

- Selma Cherchali

- Laurent Coeurdevey

- Fabien Coubard

- Myriam Cournet

- Pierre Couteron

- Benoît Deffontaines

- Jean-François Dejoux

- Jean-Marc Delvit

- Michel Deshayes

- Julie Digne

- Patrick Duthil
- Salah Er-Raki

- Mathieu Fauvel

- Delphine Fontannaz

- Laurent Gabet

- Sébastien Gadal

- Joaquim Garrabou

- Antoine Gardel

- Muriel Gasc

- Alain Giros

- Valéry Gond

- François Goulette

- Yann Gousseau

- Adrien Gressin

- Manuel Grizonnet

- David Hello

- Jean-Baptiste Henry

- Steven Hosford

- Patrick Houdry

- Jacques Iltis

- Jordi Inglada

- André Jalobeanu

- Anne Jolly

- Said Khaba

- Philippe Kubik

- Camille Kurtz

- Kamal Labassi

- Florent Lafarge

- Tania Landes

- Camille Lelong

- Maxime Lhuillier

- Marc Lointier 
- Mustapha Maatouk

- Mioara Mandea

- Rodolphe Marion

- Antoine Masse

- Nicole Mathys

- Stéphane May

- Grégoire Mercier

- Julien Michel

- Fabrice Monnier

- Jean-Michel Morel

- Simona Niculescu

- Alami Omar

- Marie-Josée Penven

- Ghislain Picard

- Antoine Pinte

- Nathalie Pisot

- Olivier Pronier

- Catherine Proy

- Christian Puech

- Franck Ranera

- Daniel Raucoules

- Olivier Regniers

- Jean-Pierre Renaud
- Emmanuel Roux

- Aurélie Sand

- Alain Sandoz

- Arnaud Sellé

- David Sheeren

- Grégoire Sigel

- Corinne Solhier

- Vaérie Soti

- Yuliya Taralbaka

- Maguelonne Teisseire

- Monique Terrier

- Khai Thami

- Lazare Tia

- Claire Tinel

- Michael Tonon

- Thierry Toutin

- Florence Tupin

- Bruno Vallet

- Emmanuelle Vaudour

- Cécile Vignolles

- Hervé Yésou

- Josiane Zerubia 